\title{
Harmonic phase-dispersion microscope with a Mach-Zehnder interferometer
}

\author{
Andrew Ahn, Changhuei Yang, Adam Wax, Gabriel Popescu, Christopher Fang-Yen, \\ Kamran Badizadegan, Ramachandra R. Dasari, and Michael S. Feld
}

\begin{abstract}
Harmonic phase-dispersion microscopy (PDM) is a new imaging technique in which contrast is provided by differences in refractive index at two harmonically related wavelengths. We report a new configuration of the harmonic phase-dispersion microscope in a Mach-Zehnder geometry as an instrument for imaging biological samples. Several improvements on the earlier design are demonstrated, including a single-pass configuration and acousto-optic modulators for generating the heterodyne signals without mechanical arm scanning. We demonstrate quantitative phase-dispersion images of test structures and biological samples. (C) 2005 Optical Society of America

OCIS codes: $120.3180,170.3880$.
\end{abstract}

\section{Introduction}

Many biological samples exhibit little absorption at visible wavelengths and can be regarded as purely phase objects. Microscopy techniques such as phase contrast and differential interference contrast perform imaging of such objects by converting sample optical path delays into differences of intensity at the detector plane..$^{1,2}$

Recently the technique of phase-dispersion microscopy (PDM) for imaging transparent biological samples was developed. ${ }^{3}$ In PDM, two beams that differ in wavelength by a factor of 2 are incident upon a Michelson interferometer, with the object of interest placed in the sample arm. One measures heterodyne signals for both wavelengths by scanning the reference arm, and the dispersion between the two wavelengths is calculated from the interferometer fringe patterns. This method was subsequently extended to imaging of three-dimensional systems, with a technique called phase-dispersion optical tomography. ${ }^{4}$ These techniques use the dispersion of biological materials as a source of contrast. Experiments showed

A. Ahn, C. Yang, A. Wax, G. Popescu, C. Fang-Yen (minwah@mit.edu), R. R. Dasari, and M. S. Feld are with the G. R. Harrison Spectroscopy Laboratory, Massachusetts Institute of Technology, Cambridge, Massachusetts 02139. K. Badizadegan is with Massachusetts General Hospital, Boston, Massachusetts 02114 .

Received 3 June 2004; revised manuscript received 20 October 2004; accepted 20 October 2004.

0003-6935/05/071188-03\$15.00/0

(C) 2005 Optical Society of America that the phase-dispersion technique has considerable potential for biomedical imaging applications. However, the instrument was slow, requiring $3 \mathrm{~h}$ for a $100 \times 100$ pixel image, and the double-pass geometry through the sample made the device difficult to align and prone to errors.

In this paper we describe a new phase-dispersion microscope based on a Mach-Zehnder interferometer and using acousto-optic frequency shifting.

\section{Experimental Setup}

The setup (Fig. 1) contains two modules: beam preparation and a Mach-Zehnder interferometer. The light source is a diode-pumped continuous-wave doubled Nd:YAG laser with output at both the fundamental $\left(\lambda_{1}=1064 \mathrm{~nm}\right)$ and the second-harmonic $\left(\lambda_{2}\right.$ $=532 \mathrm{~nm}$ ) wavelengths. The two beams are separated by dichroic mirror DCM1 and coupled into single-mode fibers by fiber couplers. At the output of the fibers the beams are collimated and are subsequently recombined by a second dichroic mirror, DCM2. The optical fibers ensure that the laser beams are nearly Gaussian in profile. In the interferometer module, beam splitter BS1 divides the two beams into sample and reference arms. In the sample arm the composite beam is focused onto the sample by a $20 \times$ microscope objective and, after passing through the sample, is recollimated by a second, identical objective. The diameters of the focused beams were 13 and $4 \mu \mathrm{m}$ for wavelengths $\lambda_{1}$ and $\lambda_{2}$, respectively, as we measured by scanning a knifeedge across the beams' foci and measuring the transmitted power with photodiodes PD1 and PD2. 


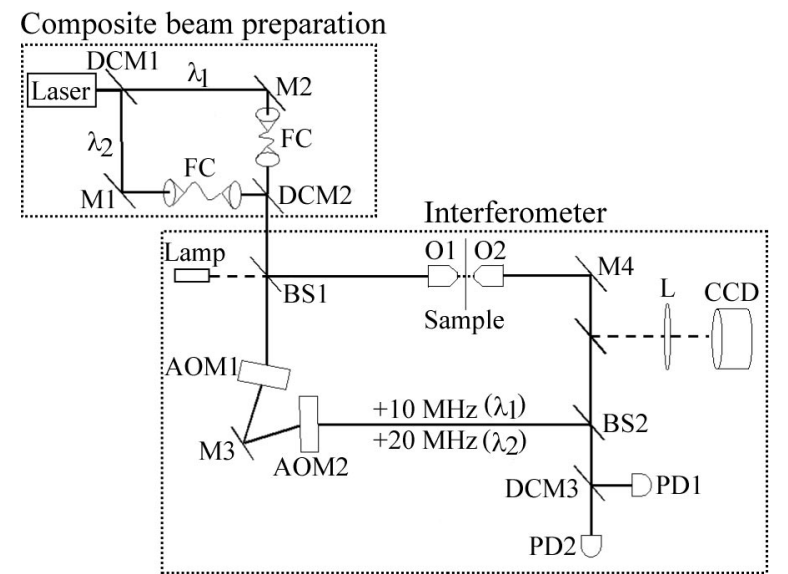

Fig. 1. Mach-Zehnder phase-dispersion microscope setup: M1M4, mirrors; FCs, fiber couplers; O1, O2, microscope objectives; other abbreviations defined in text.

A bright-field microscope (lamp, lens L, and CCD) facilitates alignment.

The sample is positioned by a three-dimensional motorized translation stage. The $z$-axis translation is used for adjusting focus, while the $x$ and $y$ axes are used to create the microscope image. To achieve frequency shifting without mechanically scanning one arm of the interferometer, we placed two acoustooptic modulators $(\mathrm{AOMs})$ in the reference arm. The crystals are aligned such that AOM1 upshifts the optical frequency of the 1064-nm beam by $110 \mathrm{MHz}$ (first-order beam) and that of the 532-nm beam by $220 \mathrm{MHz}$ (second-order beam). The second AOM downshifts the optical frequency of the 1064-nm beam by $120 \mathrm{MHz}$ (first-order beam) and that of the $532-\mathrm{nm}$ beam by $240 \mathrm{MHz}$ (second-order beam). The sample and reference arm beams are recombined by beam splitter BS2. The beams are split into component wavelengths by dichroic mirror DCM3, and accoupled photodetectors (New Focus 1801/1811) measure heterodyne signals at frequencies $\Delta \Omega$ $=10 \mathrm{MHz}$ for $\lambda_{1}=1064 \mathrm{~nm}$ and $2 \Delta \Omega=20 \mathrm{MHz}$ for $\lambda_{2}=532 \mathrm{~nm}$.

The ac portions of the photodetector signals for beams $\lambda_{1}$ and $\lambda_{2}$ are given, respectively, by

$$
\begin{aligned}
& V_{1}(t) \propto \cos \left[\Delta \Omega t+k_{1}\left(\Delta L_{1}+\delta s(t)\right],\right. \\
& V_{2}(t) \propto \cos \left[2 \Delta \Omega t+2 k_{1}\left(\Delta L_{2}+\delta s(t)\right],\right.
\end{aligned}
$$

where $k_{1}=2 \pi / \lambda_{1}, \Delta L_{1}=\Delta L_{1}{ }^{S}-\Delta L_{1}{ }^{R}$, and $\Delta L_{2}$ $=\Delta L_{2}{ }^{S}-\Delta L_{2}{ }^{R}$ are the path-length differences between signal and reference arms at wavelengths $\lambda_{1}$ and $\lambda_{2}$, and $\delta s(t)$ accounts for interferometer phase noise that is due to external perturbations, such as air motion, vibrations, and thermal drift, which is assumed to be nondispersive. To measure the phase difference between the two arms we use a frequency doubler to double the signal $V_{1}(t)$, which yields

$$
V_{1}^{d}(t) \propto \cos \left[2 \Delta \Omega t+2 k_{1}\left(\Delta L_{1}+\delta s(t)\right] .\right.
$$

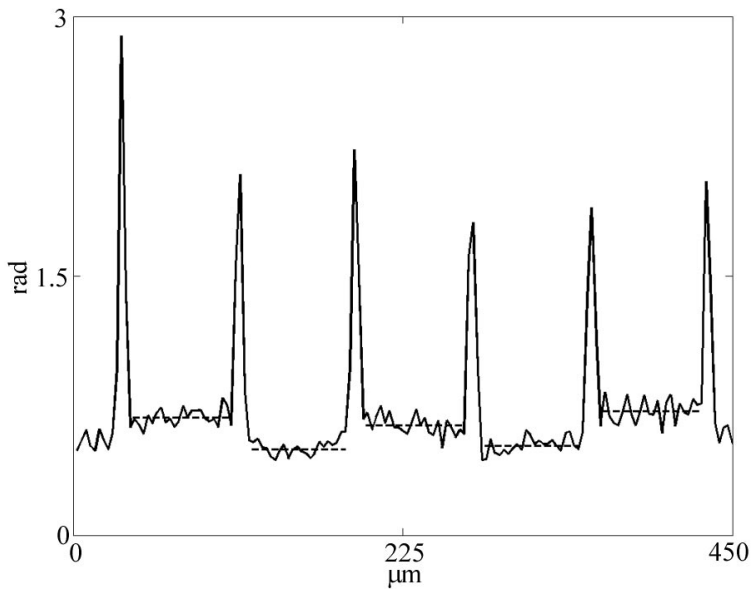

Fig. 2. Cross section of the phase image of a BK7 grating. The sharp peaks may be due to beam misalignment. Dashed lines indicate averages over regions between peaks.

Two phase-detector circuits (Analog Devices, $\mathrm{AD}$ 8302) are then used to measure the phase difference between signals $V_{1}^{d}(t)$ and $V_{2}(t)$, one measuring the in-phase quadrature and one (by means of a $90^{\circ}$ delay line) measuring the out-of-phase quadrature. The measured phase difference is $\Delta \phi=2 k_{1}\left(\Delta L_{1}-\Delta L_{2}\right)$ and, as the paths of the beams at wavelengths 1 and 2 are identical, any phase difference is due to differences in dispersion. Two-dimensional dispersion imaging is performed by in-plane scanning of the sample. As an unknown background dispersion exists as a result of the presence of various interferometer elements, all phase measurements shown here should be considered relative to other lateral points on the sample, not as absolute measurements of total sample dispersion.

The outputs of both phase detectors are digitized by a National Instruments analog-to-digital board and analyzed with LabVIEW software. An approximation in the algorithm that is used to demodulate the phase
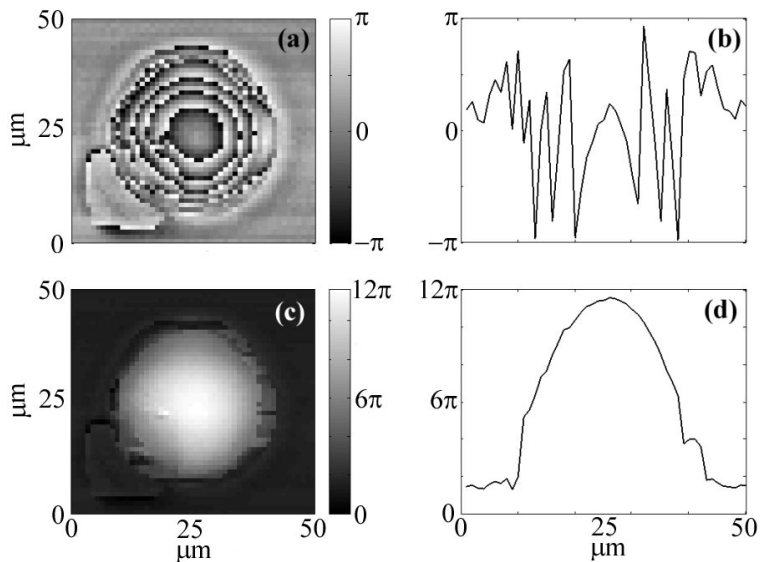

Fig. 3. (a) Phase-dispersion image of a $40-\mu \mathrm{m}$-diameter polystyrene sphere in glycerol. (b) Vertical cross section through the center of the sphere. (c) Image after the phase-unwrapping algorithm was applied. (d) Vertical cross section of the unwrapped image through the center of the sphere. 


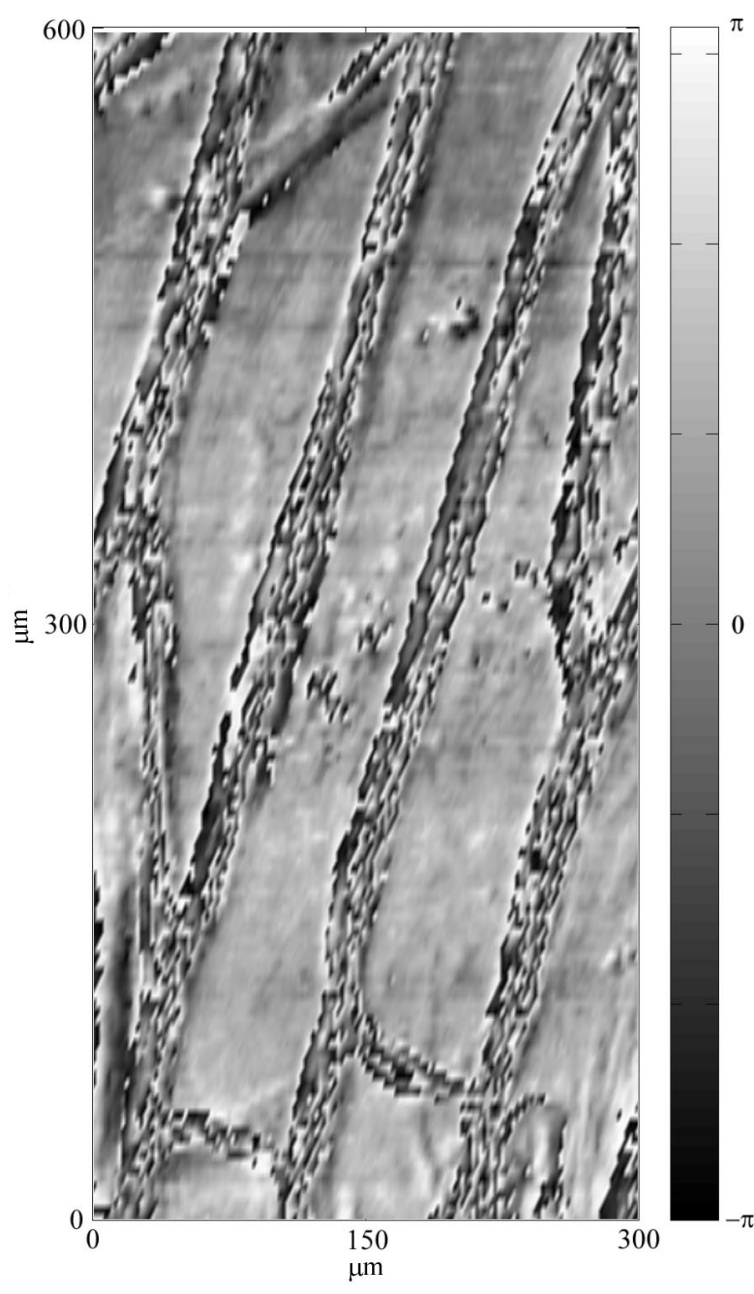

Fig. 4. Phase-dispersion image of cells of an onion skin.

detection outputs introduces an error of $\pm 0.071 \mathrm{rad}$ in the calculation. The LabVIEW program also controls the motors of the scanning stages and records data.

\section{Experimental Results}

To verify the dispersion measurements quantitatively, we obtained PDM data from a rectangular phase grating made from BK7 glass (Fig. 2). The rectangular profile had a height of $1.064 \mu \mathrm{m}$ with a $75-\mu \mathrm{m}$ pitch. The refractive indices of BK7 at 1.064- and 0.532- $\mu \mathrm{m}$ wavelengths are 1.506 and 1.519 , respectively. The PDM measurement gave $0.22 \pm 0.07 \mathrm{rad}$, in good agreement with the expected value of $0.21 \mathrm{rad}$. The spikes associated with the edges of the grooves may reflect either size mismatch of the two beams or a small lateral misalignment.

Figure 3 illustrates the phase-dispersion image retrieved from a polystyrene bead $40 \mu \mathrm{m}$ in diameter. The beads were immersed in glycerol and sandwiched between two glass coverslips. Because of the large size of the diameter relative to the wavelength, the measured phase dispersion exceeded $2 \pi$. This phasewrapping effect is illustrated by the concentric rings in Fig. 3(a); Fig. 3(c) shows the result after a numerical phase-unwrapping procedure was applied. Vertical cross sections of these images are presented in Figs. 3(b) and 3(d), respectively. The ghost image to the lower right of the bead may have been caused by stray reflections from dichroic mirror DCM1 or from the same edge effect observed in the grating image.

A PDM image of a layer of onion skin is shown in Fig. 4. The cell boundaries and substructures are resolved by our technique with high contrast.

\section{Discussion}

The configuration presented here represents an improvement over the earlier Michelson setup in a number of ways. The single-pass setup greatly simplifies the optical alignment and reduces ghosting effects from slight misalignment of the Michelson beam. Heterodyne signals are created by acousto-optic modulators instead of by piezoelectric transducer scanning of a mirror. This gives a continuous heterodyne signal, and several limitations of the a piezoelectric transducer crystal, such as hysteresis and creep, are avoided. In addition, the heterodyne frequency obtained is much higher, which reduces the sensitivity of the instrument to $1 / f$ noise and allows faster data acquisition. Moreover, phase detection is performed in hardware instead of software as in the earlier version, which both limited the heterodyne frequency and caused delays for signal processing. As a result of these improvements, images can be obtained at a rate of $50 \mathrm{~ms} /$ pixel, compared with $1000 \mathrm{~ms} /$ pixel in the earlier system. In these experiments laser intensity noise problems prevented us from achieving a much higher data acquisition rate, of the order of $1 \mathrm{~ms} /$ pixel. We intend to attempt to increase the imaging rate further and explore techniques for wholefield phase imaging.

This research was supported by the National Institutes of Health grant P41-RR02594-18 and by the Hamamatsu Corporation.

\section{References}

1. C. Yang, A. Wax, I. Georgakoudi, E. B. Hanlon, K. Badizadegan, R. R. Dasari, and M. S. Feld, "Interferometric phase-dispersion microscopy," Opt. Lett. 25, 1526-1528 (2000), and references therein.

2. F. Zernike, "How I discovered phase contrast," Science 121, 345-349 (1955).

3. M. Bass, ed., Handbook of Optics (McGraw-Hill, New York, 1995), Vol. 2, Chap. 17.

4. C. Yang, A. Wax, R. R. Dasari, and M. S. Feld, "Phasedispersion optical tomography," Opt. Lett. 26, 686-688 (2001). 